\title{
A Probability Model Based on Frequency Quadrilaterals for Travelling Salesman Problem
}

\author{
Peter Yong Wang* \\ No.2, Beinong Road, Huilongguan, Changping, North China Electric Power University, Beijing 102206, China \\ ${ }^{*}$ Corresponding author
}

\begin{abstract}
The research aims to generate a sparse graph for travelling salesman problem so as to reduce its complexity. A probability model based on frequency quadrilaterals is given to show that the average frequency of an edge is $3 N$ if its total frequency is computed with $N$ random frequency quadrilaterals in complete graph $K_{n}$. For an edge in the optimal Hamiltonian cycle, the minimum frequency is derived as $\left(3+\frac{2}{n-2}\right) N$.It suggests we can throw away the edges with frequency below $\left(3+\frac{2}{n-2}\right) N$ and still keep the optimal Hamiltonian cycle in the reserved graph. We test the probability model with quitea few Euclidean TSP instances. The results show the threshold $(3+$ $\left.\frac{2}{n-2}\right) N$ is too small for most of the instances.
\end{abstract}

Keywords-travelling salesman problem; sparse graph; probability model; frequency quadrilateral; optimal $i$-vertex path

\section{INTRODUCTION}

Given a tourist map with $n$ cities $\{1,2, \cdots, n\}$ and the distance function $d(u, v)>0$ for any two different cities $u, v \in\{1,2, \cdots, n\}$, a salesman expects to find the shortest cycle, namely the optimal Hamiltonian cycle $(\mathrm{OHC})$, that visits each city once and exactly once. This is the origination of traveling salesman problem (TSP). A cycle visiting each of the cities exactly once is a Hamiltonian cycle $(H C)$ which is noted as $H=\left(v_{1}, v_{2}, \cdots, v_{n}, v_{1}\right)$ where $v_{k} \in\{1,2, \cdots, n\}(1 \leq k \leq n)$. The distance of an $H C$ is computed as $d(H C)=d\left(v_{1}, v_{n}\right)+$ $\sum_{k=1}^{n-1} d\left(v_{k}, v_{k+1}\right)$. The objective of TSP is to find the $O H C$ with the minimum distance. Due to its theoretical values to many $N P$-hard problems and wide applications in engineering, TSP has been extensively studied in the fields of combinatorial optimization, graph theory and computer science in order to resolve it within an acceptable computation time [1].

TSP has been proven to be $N P$-complete [2] in decision theory, i.e., there is no polynomial-time algorithms to resolve $T S P$ unless $P=N P$. In graph theory, TSP is represented as a complete weighted graph $K_{n}$. The cities are mapped into vertices set $V=\left\{v_{1}, v_{2}, \cdots, v_{n}\right\}$ and the routes between each two cities are noted as edges $(u, v) \in E$ for $u, v \in$ $\left\{v_{1}, v_{2}, \cdots, v_{n}\right\}$ and $u \neq v$. Without loss of generality, $|E|=\left(\begin{array}{l}n \\ 2\end{array}\right)$. For the symmetrical TSP, $d(u, v)=d(v, u)$ for any pair of vertices $u, v$. The efficient algorithms, such as the exact and approximate algorithms, are designed to detect the $O H C$ in $K_{n}$. However, the time complexity of the exact algorithms is superpolynomial $O\left(a^{n}\right)$ [3-4], where $a>1$. Considering more constraints, the integer programming methods, such as the branch and bound [5, 6] or cutting plane [7, 8],are able to resolve TSP with thousands of cities. In 2006, a VLSI application with 85,900 vertices has been resolved with the improved cutting plane [8] with a 128-node computer system.

On the other hand, the computation time of the approximate algorithms and heuristics are greatly reduced [9]. For example, the MST heuristics [10] and Chris to fides' algorithms [11] are able to find an approximation in time $O\left(n^{2}\right)$ and $O\left(n^{3}\right)$ for metric TSP, respectively. The LKH is taken as one of the competitive tourimprovement algorithms for TSP[12]. However, these approximate algorithms and heuristics cannot guarantee to find the $\mathrm{OHC}$ within a polynomial time.

Some other scholars try to design the TSP algorithms based on a sparse graph. The sparse graphs include a small number of edges and the search space of the $O H C$ will drop sharply. The number of $H C$ s in a sparse graph of average degree $d$ is less than $e^{*}\left(\frac{d}{2}\right)^{n}$ owing to the work of Sharir\& Welzl [13], where $e^{*}$ is the base of natural logarithm. Heidi [14] gave the lower bound of the number of $H C$ s roughly as $\left(\frac{d}{2}\right)^{n}$ in a sparse graph of average degree $d$. Björklund [15] proved that TSP with bounded degree can be resolved in time $O\left((2-\varepsilon)^{\mathrm{n}}\right)$, where $\varepsilon$ relies on the maximum degree of vertex rather than the scale of $T S P n$. In addition, Glencora, Erik and Siamak[16] presented a polynomial-time approximation schemes for general boundedgenus graphs. In the case of asymmetric TSP, Shayan and Amin [17] proved a constant factor approximation algorithms for the bounded-genus graphs and the constant factor is $22.5 \times(1+1 / n)$ for planar TSP. The recent research explains that the hardness of $T S P$ will be reduced to some extent if we use a sparse graph for TSP.

In previous research [18-20], we computed the frequency graphs for TSP where the frequency of the edges in the $O H C$ is much bigger than that of most of the other edges. It enlightens us to derive a sparse graph based on the frequency graphs. But the lower bound of the frequency of the $O H C$ edges is not given. This paper computes the lower bound of the frequency of the $O H C$ edges using a probability model based on frequency quadrilaterals. When we compute the frequency of an $\mathrm{OHC}$ edge with $\mathrm{N}$ random frequency quadrilaterals with it, the minimum frequency of the $O H C$ edge is derived as $\left(3+\frac{2}{n-2}\right) N$. It is bigger than the average frequency $3 N$ of a common edge. Therefore, it is necessary to choose the edges with frequency above $3 N$ to compose another graph which is sparser than the complete graph for TSP. 
The rest of the paper is organized as follows. In section 2, the optimal i-vertex path is briefly introduced to compute the frequency quadrilaterals. Based on the frequency quadrilaterals, a probability model for the $O H C$ edges is presented in sections 3.The experiments to test the probability model are executed in section 4 . The conclusion is given in the last section.

\section{THE FREQUENCY QUADRILATERAL}

We start with the optimal paths to deduce the frequency quadrilaterals. Given $i$ vertices $\left\{v_{1}, v_{2}, \cdots, v_{i}\right\}(1 \leq i \leq n)$ in $K_{n}$, a path $P$ composed of the $i$ vertices is noted as $P^{i}=$ $\left(v_{1}, v_{2}, \cdots, v_{i}\right)$ and its distance is $d\left(P^{i}\right)=\sum_{k=1}^{i-1} d\left(v_{k}, v_{k+1}\right)$. The vertices $v_{1}$ and $v_{\mathrm{i}}$ on both ends are the end vertices. The other vertices between $v_{1}$ and $v_{\mathrm{i}}$ are the intermediate vertices. If we take $v_{1}$ and $v_{\mathrm{i}}$ as the two end vertices, there are $(n-2)$ ! such $P^{i}$ s composed of the $i$ vertices. Among the $(n-2) ! P^{i} \mathrm{~s}$, there is one path which has the minimum distance $d\left(P^{i}\right)$. We name this path as the optimal path $\left(O P^{i}\right)$. Since we have $\left(\begin{array}{l}i \\ 2\end{array}\right)$ ways to pick two end vertices from $\left\{v_{1}, v_{2}, \cdots, v_{i}\right\}$, there are $\left(\begin{array}{l}i \\ 2\end{array}\right) O P^{i}$ s arising from the $i$ vertices $\left\{v_{1}, v_{2}, \cdots, v_{i}\right\}$. In $K_{n}$, there are total $\left(\begin{array}{c}i \\ 2\end{array}\right)\left(\begin{array}{l}n \\ i\end{array}\right) O P^{i} s[18]$. For every edge $(u, v)$ in $K_{n}$, there are $\left(\begin{array}{c}n-2 \\ i-2\end{array}\right) O P^{i}$ s with $(u, v)$ in average.

Let's analyze the combinatorics and properties of the $P^{i} \mathrm{~s}$ and the $O P^{i}$ s for symmetric TSP. Firstly, the number of $P^{i} \mathrm{~s}$ is $\frac{i !}{2}\left(\begin{array}{l}n \\ i\end{array}\right)$ whereas that of the $O P^{i} \mathrm{~s}$ is $\left(\begin{array}{l}i \\ 2\end{array}\right)\left(\begin{array}{l}n \\ i\end{array}\right)$. When $i \geq 4$, the number of $O P^{i}$ s is much smaller than that of $P^{i}$ s. Secondly, the distance of every $O P^{i}$ is minimum among the $(n-2) ! P^{i}$ s. They cannot become shorter whatever their middle vertices are exchanged. This is a special property as the optimal Hamiltonian path and the $O H C$. Hence, our intuition is that the $O P^{i}$ s have many intersections with the $O H C$. The intersections between the $O P^{i} \mathrm{~s}$ and the $O H C$ are the $O P^{j} \mathrm{~s}(j<i)$ with a smaller number of edges. When $j=2$, the intersections are actually the edges. We take the number of times that an edge $(u, v)$ appears in all of the $O P^{i}$ s as its frequency $F(u, v)$, i.e., the number of $O P^{i}$ s with $(u, v)$. In general, the $F(u, v)$ of an edge $(u, v) \in O H C$ will be much bigger than that of most of the other edges. This fact has been verified with a number of real-world TSP instances [18-20].

After we compute the frequency of every edge from the $O P^{i} \mathrm{~s}$, a frequency graph $(F G)$ is obtained. An $F G$ has the same topological structure as a weighted graph $(W G)$. The difference between them is the values on the edges. The distances in $W G$ represent the metric distance, time or cost etc. in various backgrounds. Each distance $d(u, v)$ reflects the importance of the corresponding edge $(u, v)$. However, it cannot reflect the common property of the special structures with it. After a $W G$ is converted into an $F G$ with a set of special structures, such as $O P^{\mathrm{i}}$ s, with the common property, the frequency of edges will intensively show their common property. For example, the common property of the $O P^{\mathrm{i}} \mathrm{s}$ is that they have the minimum distance. When the $O P^{\mathrm{i}} \mathrm{s}$ are used to compute the $F G$ s, the frequency of the edges in the $O H C$ will be much bigger than that of most of the other edges.

In structures, the $O P^{\mathrm{i}} \mathrm{s}$ have many intersections of edges with the $O H C$. We puzzled how big the frequency of the $O H C$ edges will be after they are enumerated from all of the $O P^{\mathrm{i}} \mathrm{s}$. For any edge in an $O P^{i}$, its frequency is equal to 1 . Thus we cannot distinguish the $i$ - 1 edges according to their frequency in view of an $O P$. It is desirable to obtain another kind of structures in which the frequency of edges is different, especially where the frequency of the $\mathrm{OHC}$ edges is different from that of the other edges in most cases. Then the frequency of the $O H C$ edges will be precisely estimated when an $F G$ is computed with such structures. Such structures are the i-vertex frequency polygons. Each i-vertex frequency polygon is computed with the $\left(\begin{array}{l}i \\ 2\end{array}\right) O P^{\mathrm{i}} \mathrm{s}$ in the corresponding i-vertex weighted polygon. Among the $\left(\begin{array}{l}i \\ 2\end{array}\right) O P^{\mathrm{i}} \mathrm{s}, i O P^{\mathrm{i}} \mathrm{s}$ belong to local optimum of the i-vertex polygon. The minimum frequency of the edges in the local optimum is at least $i-1$ in the $\mathrm{i}$-vertex frequency polygon. Except for the $i O P^{\mathrm{i}} \mathrm{s}$ in the local optimum, we assume the $\left(\begin{array}{l}i \\ 2\end{array}\right)$ edges are uniformly distributed in the other $\frac{i(i-3)}{2} O P^{\mathrm{i}} \mathrm{s}$. The average frequency of the edges in the local optimum is computed as $2 i-4$ whereas that of the other edges is $i$-3. It says the frequency of the edges in the local optimum will be bigger than the average frequency $i-1$ of all of edges in case that the frequency polygon includes $\left(\begin{array}{l}i \\ 2\end{array}\right)$ edges, where $i \geq 4$. On the other hand, the frequency of the other edges will be smaller than the average frequency $i-1$ of all of edges.

The $F G$ computed with the i-vertex frequency polygons has the same function as those computed with the $O P^{\mathrm{i}}$ s. Different from the $O P^{\mathrm{i}} \mathrm{s}$, the frequency of edges in the i-vertex frequency polygons have much difference. If we select $\mathrm{Ni}$-vertex frequency polygons with an edge $(u, v) \in O H C$ to compute its total frequency, the frequency of $(u, v)$ is noted as $F(u, v)=$ $\sum_{k=1}^{N} f_{k}$ and $f_{\mathrm{k}}$ is the frequency of $(u, v)$ in the $k$ th frequency polygons. If $f_{\mathrm{k}}$ is equal to the frequency of the edges in the local optimum in most of the frequency polygons, the frequency of the $\mathrm{OHC}$ edges can be precisely estimated. It will consume a lot of time to compute the i-vertex frequency polygons if $i$ is big. To reduce the complexity, we first compute the frequency of edges with the frequency quadrilaterals. In the following, we first compute a frequency quadrilateral for a weighted quadrilateral and then analyze the probability of the frequency of edges in the frequency quadrilaterals.

A weighted quadrilateral $K_{4}$ is shown in Fig. 1 (a). It is composed of four vertices $\mathrm{A}, \mathrm{B}, \mathrm{C}$ and $\mathrm{D}$. The real numbers aside the edges are distances of edges. The distances of edges are different to compute the $\left(\begin{array}{l}4 \\ 2\end{array}\right)=6 O P^{4}$ s with the four-vertex and three-line inequality [20]. In Fig.1, the inequality array based on the distances of the six edges is derived as $d(A, C)+$ $d(B, D)>d(A, B)+d(C, D)>d(A, D)+d(B, C) \quad$ (The middle edges are eliminated for symmetric TSP). The six $O P^{4} \mathrm{~s}$ computed with the three inequalities are $(\mathrm{D}, \mathrm{A}, \mathrm{B}, \mathrm{C}),(\mathrm{D}, \mathrm{A}, \mathrm{C}$, B), (B, A, D, C), (A, B, C, D), (C, B, D, A) and (B, C, D, A). The edges and their frequency enumerated from the six $O P^{4} \mathrm{~S}$ compose a frequency quadrilateral shown in Fig. 1 (b). The four edges (A, D), (B, C) and (A, B), (C, D) with frequency 5 and 3 are included in the local optimum. We discovered that it is the sum distance of the three pairs of non-adjacent edges which determines the edges' frequency. For each pair of nonadjacent edges, the bigger their sum distance is, the smaller their frequency will be. For example in Fig. 1 (a), the three 
pairs of non-adjacent edges are (A, D) \& (B, C), (A, B) \& (C, D) and $(\mathrm{A}, \mathrm{C})$ \& $(\mathrm{B}, \mathrm{D})$. Their sum distances are 4.0, 7.5 and 13.0, respectively. On the contrary, the frequency of the three pair of non-adjacent edges is 5,3 and 1 , respectively.

Given a weighted quadrilateral with $6 O P^{4} \mathrm{~s}$, its frequency quadrilateral is unique. In a frequency quadrilateral $A B C D$, the frequency of every edge is 5,3 or 1 . Moreover, the two nonadjacent edges have the identical frequency. The frequency of

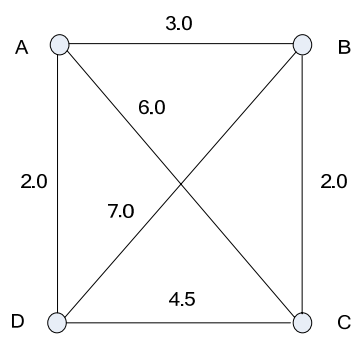

(a)

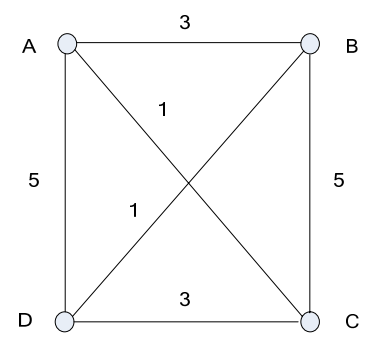

(b)
FIGURE I. A WEIGHTED QUADRILATERAL(A) AND ITS FREQUENCY QUADRILATERAL (B)

\section{THE PROBABILITY MODEL}

Given an edge $(u, v)$ in $K_{n}$, it is included in $\left(\begin{array}{c}n-2 \\ 2\end{array}\right)$ weighted quadrilaterals. Meanwhile, we have the corresponding $\left(\begin{array}{c}n-2 \\ 2\end{array}\right)$ frequency quadrilaterals. In each of the frequency quadrilaterals, its frequency $f$ may be 5,3 or 1 . Based on the analysis at the end of section 3 , we assign the frequency $f=5,3$ and 1 of $(u, v)$ with the identical probability in each of the frequency quadrilaterals.We use $p_{5}, p_{3}$ and $p_{1}$ denote the probability that $(u, v)$ has $f=5,3$ or 1 , then $p_{5}=p_{3}=p_{1}=\frac{1}{3}$ for edge $(u, v)$. If we select $N$ quadrilaterals with $(u, v)$ to compute its total frequency, the expected frequency is $F(u, v)=\frac{5+3+1}{3} N=3 N$. It is the average case for all of the edges.

For the edges in the $O H C$, there are some special quadrilaterals where their frequency is either 5 or 3 , such as the quadrilaterals shown in Fig. 2. The boldface lines are the edges in the OHC. For $(u, v) \in O H C$, there are $n-5(n \geq 6)$ quadrilaterals composed of $(u, v)$ and its $n$-5 further nonadjacent edges, such as the quadrilateral composed of vertices $0,1, u, v$. There are two quadrilaterals composed of $(u, v)$ and its two near non-adjacent edges, such as the quadrilaterals $i-1, i, u, v$ and $u, v, j, j+1$. Among the $n-3$ frequency quadrilaterals, the frequency of $(u, v)$ is at least 3 . Otherwise, the two edges $(u, v)$ and its non-adjacent edge will be replaced by the other two non-adjacent edges. In $K_{n}$, the total number of frequency quadrilaterals with $(u, v)$ is $\left(\begin{array}{c}n-2 \\ 2\end{array}\right)$. If we assign $f=5$ and 3 of $(u, v) \in O H C$ with the identical probability in the $n$-3 quadrilaterals, the probability that $(u, v) \in O H C$ has $f=5$ and 3 will be computed as $p_{5}=p_{3}=\frac{1}{3}+\frac{1}{3(n-2)}$ and then $p_{1}(f=$ 1) $=\frac{1}{3}-\frac{2}{3(n-2)}$. The probability model is given as formula (1). the six edges composes a stable frequency space $\{5,3,1\}$. In addition, each of the frequency 5, 3 and 1 for every edge will appear twice based on the orders of $d(A, C)+d(B, D), d(A, B)+d(C, D)$ and $d(A, D)+d(B, C)$. It means every edge will have two chances to have frequency 5 or 3 or 1 . Therefore we can give a probability model for the frequency 5,3 and 1 of an edge based on the frequency quadrilaterals.

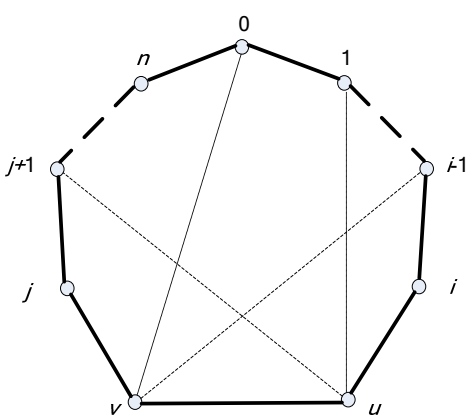

FIGURE II. THE QUADRILATERALSCOMPOSED OF ANEDGE $(\mathrm{U}, \mathrm{V}) \in$ OHCANDTHE OTHER OHCEDGES

$$
\left\{\begin{array}{l}
p_{5}(f=5)=\frac{1}{3}+\frac{1}{3(n-2)} \\
p_{3}(f=3)=\frac{1}{3}+\frac{1}{3(n-2)} \\
p_{1}(f=1)=\frac{1}{3}-\frac{2}{3(n-2)}
\end{array}\right.
$$

If we select $N$ frequency quadrilaterals with $(u, v) \in O H C$, the total frequency is computed as $F((u, v) \in O H C)=$ $\left(3+\frac{2}{n-2}\right) N>3 N$. It means we can choose the edges with $F$ above $3 N$ to compose a sparse graph for TSP. In applications, we throw away the edges $(u, v)$ with $F(u, v)<\left(3+\frac{2}{n-2}\right) N$ to generate the sparse graph. Then the TSP complexity will be reduced to some extent based on the sparse graph.

\section{EXAMPLES AND ANALYSIS}

We test the probability model with some Euclidean TSP instances in TSPLIB [21]. First we select $N=\frac{1}{2}\left(\begin{array}{c}n-2 \\ 2\end{array}\right)$ weighted quadrilaterals with an edge $(u, v)$ at random. Then the total frequency $F(u, v)$ is computed with the algorithm in Figure 3. At last $\left(3+\frac{2}{n-2}\right) N$ is taken as the threshold to generate a sparse graph for these TSP instances (the edges with $F(u, v)$ above $\left(3+\frac{2}{n-2}\right) N$ are reserved).

To verify the rightness of the probability model, the $O H C$ of these TSP instances is computed first with the Concorde package online (NEOS Server for Concorde) [22]. The minimum total frequency $F_{\text {min }}$ of the edge $(u, v) \in O H C$ is recorded for comparisons with the value $\left(3+\frac{2}{n-2}\right) N$. We also use the minimum $F_{\min }$ as a second threshold to compute the second sparse graph. The two sparse graphs are compared. For convenience, we use $f$ and $f_{\min }$ to note $3+\frac{2}{n-2}$ and $\frac{F_{\min }}{N}$, respectively. That's $f=\frac{F}{N}=3+\frac{2}{n-2}$ and $f_{\min }=\frac{F_{\min }}{N}$. If the 
sparse graph includes $K$ edges, a ratio $r=\frac{K}{\left(\begin{array}{c}n \\ 2\end{array}\right)}$ is defined to represent the sparsity of the reserved graph. The smaller the $r$, the sparser the reserved graph is. We do 10 experiments for every TSP instance and the average results are computed and given in Table 1.

TABLE I. THE RESULTS COMPUTED WITH RANDOM FREQUENCY QUADRILATERALS

\begin{tabular}{lclccc}
\hline \multicolumn{1}{c}{$\boldsymbol{T S P}$} & $\boldsymbol{n}$ & \multicolumn{1}{c}{$\boldsymbol{f}$} & $\boldsymbol{f}_{\min }$ & $\boldsymbol{r}(\boldsymbol{f})$ & $\boldsymbol{r}\left(\boldsymbol{f}_{\min }\right)$ \\
\hline pr144 & 144 & 3.014085 & 3.067706 & 0.428344 & 0.418933 \\
pr226 & 226 & 3.008929 & 3.367114 & 0.422025 & 0.307688 \\
kroA200 & 200 & 3.0101019 & 3.613993 & 0.431800 & 0.217503 \\
Fl417 & 417 & 3.0048199 & 3.508958 & 0.408189 & 0.203798 \\
lin318 & 318 & 3.0063299 & 3.744377 & 0.437812 & 0.185066 \\
rd400 & 400 & 3.0050259 & 3.871571 & 0.443200 & 0.155720 \\
d657 & 657 & 3.0030539 & 3.866899 & 0.444892 & 0.150856 \\
pr439 & 439 & 3.004577 & 3.909141 & 0.443866 & 0.141733 \\
rat575 & 575 & 3.00349 & 3.889385 & 0.451597 & 0.134806 \\
d493 & 493 & 3.004073 & 3.879436 & 0.457127 & 0.132910 \\
rat783 & 783 & 3.002561 & 3.925740 & 0.456193 & 0.116713 \\
u724 & 724 & 3.00277 & 3.974198 & 0.447539 & 0.115611 \\
\hline
\end{tabular}

We see $f=3+\frac{2}{n-2}$ becomes smaller according to $n$ whereas $f_{\text {min }}=\frac{F_{\min }}{N}$ increases according to $n$. It means the difference $F_{\min }-\left(3+\frac{2}{n-2}\right)$ Narises according to $n$. Therefore, the $O H C$ will be maintained in these sparse graphs if $(3+$ $\left.\frac{2}{n-2}\right) N$ is taken as the frequency threshold. When $\left(3+\frac{2}{n-2}\right) N$ is taken as the frequency threshold, $r(f)$ is less than 0.5 for these $T S P$ instances. It says less than $\frac{n(n-1)}{4}$ edges are reserved. If we use the actual $F_{\min }$ as the threshold to compute the sparse graphs, $r\left(f_{\min }\right)$ is much smaller in most cases. For example rat783 and $\mathrm{u} 724$, less than $12 \%$ of the total edges are maintained in their sparse graphs. It means the $\left(3+\frac{2}{n-2}\right) N$ is too small as a threshold to compute the sparse graph. An even sparser graph will be computed with the actual $F_{\min }$ as the frequency threshold.

\section{CONCLUSIONS}

A probability model based on frequency quadrilaterals is presented for TSP. If we select $N$ random frequency quadrilaterals with each edge to compute their frequency, the minimum frequency of the $O H C$ edge is derived as $(3+$ $\left.\frac{2}{n-2}\right) N$. Therefore we can throw away the edges with frequency below $\left(3+\frac{2}{n-2}\right) N$ to generate a sparse graph for TSP. The experiments show that for most Euclidean TSP the minimum frequency $F_{\min }$ of the $O H C$ edge is much bigger than $(3+$ $\left.\frac{2}{n-2}\right) N$. We should choose a bigger threshold to compute the sparse graph for TSP. An exact frequency threshold based on the probability model will be explored in the next stage. Another question is that how many edges will be maintained in the sparse graph if we use the exact frequency threshold.

\section{ACKNOWLEDGMENT}

The authors acknowledge Hans Mittelmannet al. who created the online Concorde and the researchers who provide the TSPLIB. The authors acknowledge the Fundamental Research Funds for the Central Universities (No.2015ZD10) and the funds supported by NSFC (No.51205129).

\section{REFERENCES}

[1] D.S.Johnson, L.A.McGeoch, The traveling salesman problem and its variations, Combinatorial Optimization.Springer Press: London, 2004, vol.12, pp. 445-487.

[2] R. Karp, “On the computational complexity of combinatorial problems,” Networks(USA), vol.5(1) , pp. 45-68, 1975

[3] M. Held and R. Karp,“A dynamic programming approach to sequencing problems,” J. Sco. Indust. Appl. Math., vol.10(1) , pp. 196-210, 1962.

[4] R. Bellman, "Dynamicprogramming treatment of the travelling salesman problem, Journal of the ACM, vol.9 (1) , pp. 61-63, 1962.

[5] G. Carpaneto, M. Dell'Amico, P. Toth,"Exact solution of large-scale, asymmetric traveling salesman problems," ACMTransactions on Mathematical Software (TOMS), vol.21(4), pp. 394-409, 1995.

[6] E.D. Klerk and C. Dobre,"A comparison of lower bounds for the symmetric circulant traveling salesman problem,” Discrete Applied Mathematics, vol.159(16), pp. 1815-1826, 2011.

[7] M.S.Levine,"Finding the right cutting planes for the TSP. Journal of Experimental Algorithmics (JEA), vol.5(6) , pp. 1-16, 2000.

[8] D.Applegate, R. Bixby,V.Chvátal, W.Cook, D.G. Espinoza, M.Goycoolea \&K.Helsgaun, "Certification of an optimal TSP tour through 85900 cities,” Operations Reearch Letters, vol. 37(1) , pp. 11-15, 2009.

[9] Y.H. Liu, "Diversified local search strategy under scatter search framework for the probabilistic traveling salesman problem,” European Journal of Operational Research, vol.191(2) , pp. 332-346, 2008.

[10] H. C. Thomas, E.L. Charles, L.R. Ronald, S. Clifford, Introduction to Algorithms, second edition. Beijing: China Machine Press, 2006, pp.636-639.

[11] J.A. Hoogeveen, “Analysis of Christofides' heuristic: Some paths are more difficult than cycles,” Operations Research Letters, vol.10(5) , pp. 291-295, 1991.

[12] K. Helsgaun, "An effective implementation of the Lin-Kernighan traveling salesman heuristic,” 2012. Available: www2.iwr.uniheidelberg.de/groups/comopt/ software/TSPLIB95/tsp/.

[13] M. Sharir, E.Welzl, “On the number of crossing-free matchings, cycles, and partitions,” SIAM J. Comput., vol.36(3) , pp. 695-720, 2006.

[14] G.Heidi,"On thenumber of Hamilton cycles in bounded degree graphs,”ETH Zurich, Switzerland, July, 2007.

[15] A. Björklund, T. Husfeldt, P. Kaski \& M. Koivisto,“The traveling salesman problem in bounded degree graphs," ACM Transitions on Algorithms, vol.8(2): , pp. 18, 2012.

[16] G. Borradaile, E.D. Demaine (MIT), S. Tazari,"Polynomial-Time Approximation Schemes for Subset-Connectivity Problems in BoundedGenus Graphs,” http://arxiv.org/abs/0902.1043

[17] S. O. Gharan, A. Saberi, “The asymmetric traveling salesman problem on graphs with bounded genus,"SODA '11 Proceedings of the twentysecond annual ACM-SIAM symposium on Discrete Algorithms. San Francisco, CA, USA, Jan. 23-25, pp.967-975.

[18] Y. Wang,"The Frequency Graph for the Traveling Salesman Problem,” ICECECE 2012, Bali, Indonesia, Oct. 24-25, 2012, pp.1019-1022.

[19] Y. Wang,“A representation model for TSP,” 15th IEEE International Conference on High Performance Computing and Communications, HPCC 2013 and 11th IEEE/IFIP International Conference on Embedded and Ubiquitous Computing, EUC 2013. Zhangjiajie, China, Nov.13-15, 2013, pp.204-209.

[20] Y. Wang,“An approximate method to compute a sparse graph for traveling salesman problem," Expert Systems with Applications, vol.28(3) , pp. 1109-1118, 2015.

[21] http://comopt.ifi.uni-heidelberg.de/software/TSPLIB95/

[22] http://neos.mcs.anl.gov/neos/solvers/co:concorde/TSP.html 